\title{
Orlistat and the risk of acute liver injury: self controlled case series study in UK Clinical Practice Research Datalink
}

\author{
(c) (1) (8) OPEN ACCESS
}

\begin{abstract}
Ian J Douglas lecturer in pharmacoepidemiology, Julia Langham lecturer, Krishnan Bhaskaran lecturer in statistical epidemiology, Ruth Brauer research student, Liam Smeeth professor of clinical epidemiology
\end{abstract}

Faculty of Epidemiology and Population Health, London School of Hygiene and Tropical Medicine, London WC1E 7HT, UK

\begin{abstract}
Objective To measure the association between orlistat and acute liver injury.

Design Self controlled case series study.

Setting Population based primary care setting, United Kingdom.

Participants 94695 patients receiving orlistat and registered in the UK Clinical Practice Research Datalink and linked with Hospital Episode Statistics data between 1999 and 2011.
\end{abstract}

Main outcome measure Relative incidence of acute liver injury comparing periods when patients were receiving orlistat with periods of non-usage.

Results Among 94695 patients who received orlistat, 988 cases of acute liver injury were identified, with 335 confirmed as definite cases and 653 as probable cases. For all cases an increased incidence of liver injury was detected during the 90 day period before orlistat was first started, with an incidence rate ratio of 1.50 (95\% confidence interval 1.10 to 2.06$)$. The incidence remained raised during the first 30 days of treatment $(2.21,1.43$ to 3.42$)$, before returning to baseline levels with prolonged treatment. When the risk during the first 90 days of treatment was compared with the 90 days preceding first treatment, the incidence of liver injury was not increased (1.02, 0.67 to 1.56). An analysis restricted to definite cases showed no evidence of an increased risk of liver injury during treatment.

Conclusion The incidence of acute liver injury was higher in the periods both immediately before and immediately after the start of orlistat treatment. This suggests that the observed increased risks of liver injury linked to the start of treatment may reflect changes in health status associated with the decision to begin treatment rather than any causal effect of the drug.

\section{Introduction}

Orlistat (Xenical; Roche) is used in the treatment of obesity and was approved by the European Commission in 1998. It is a selective inhibitor of gastric and pancreatic lipase and leads to a reduction in dietary fat lipolysis and absorption. ${ }^{1}$ Although mild but unpleasant gastrointestinal side effects are commonly reported with orlistat use there is also concern that it may be associated with an increased risk of serious hepatic events. The US Food and Drug Administration first issued a warning about a possible link between orlistat and serious liver injury in $2009^{23}$ based on an analysis of 32 case reports received between 1999 and 2008. The European Medicines Agency has also reviewed the strength of evidence relating to this possible risk. ${ }^{4}$ The analysis of individual case reports is often unable to give reliable conclusions about causality and a range of population based studies are needed to help inform decisions about the likely risks and benefits of orlistat. A meta-analysis of clinical trial data involving around 10000 patients found no evidence that orlistat was associated with increases in selected variables of liver function, ${ }^{5}$ and a likely mechanism of action has not been identified. Assessments by both agencies concluded that there was no strong evidence to determine a causal association. However, results from clinical trials do not always reflect the effects of drugs when used in the general population, and to date no studies have been published based on orlistat use in the real world. Orlistat remains the only drug treatment option for obesity, is widely used, and in many countries is available without prescription. Further clarity on safety is therefore needed.

People using orlistat are likely to have a different underlying risk of acute liver events from non-users owing to obesity and its comorbidities, and many study designs would be unable to fully account for these important differences. We conducted a self controlled case series study in which each participant acts as his or her own control to avoid the inherent biases caused by differences between people. 


\section{Methods}

We carried out a population based study using the UK Clinical Practice Research Datalink (formerly the General Practice Research Database) linked with Hospital Episode Statistics data to provide a more formal assessment of any association between orlistat and adverse liver events.

\section{The Clinical Practice Research Datalink}

The Clinical Practice Research Datalink contains anonymised information on over 11 million patients registered at over 600 general practices in the United Kingdom. ${ }^{67}$ Information is continuously recorded for each patient, including a record of each consultation, diagnoses, prescribed medicines, and basic demographic data. The geographical distribution and size of general practices represented in Clinical Practice Research Datalink is largely representative of the population of England and Wales, and people registered on the database are representative of the UK population in terms of age and sex. ${ }^{8}$ The quality of data held is subject to rigorous checks and regular audits and the data have been used to conduct over 600 peer reviewed published studies. The information obtained from the database is entirely anonymised.

\section{Hospital episode statistics}

Hospital episode statistics is a database containing information on all admissions to National Health Service hospitals in England (including private patients treated in NHS hospitals). ${ }^{9}$ Clinical information relating to diagnoses and procedures carried out during each hospital stay are recorded using ICD-10 codes (international classification of diseases, 10th revision). Around half of the active practices contributing to the Clinical Practice Research Datalink have been linked with Hospital Episode Statistics, thus providing additional clinical information for all patients from these practices who were admitted to hospital between 1997 and 2010.

\section{Study design}

We carried out a self controlled case series analysis. This study design is derived from rate modelling using a Poisson distribution and is comparable with cohort methodology. It relies on within person comparisons in a population with both the outcome and exposure of interest. ${ }^{10}{ }^{11}$ Incidence rate ratios are derived, comparing the rate of events during exposed periods with that during all other observed periods. A major advantage of this design is that it removes the potential confounding effect of both recorded and unrecorded characteristics that vary between people but are fixed over time within people. Age, which varies over time, is adjusted for in the analysis.

The method relies on three key assumptions. Firstly, recurrent events must be independent - that is, the likelihood of a second event is not influenced by having a first event. Although this may not be true for liver injury, a convenient way to avoid bias is to consider only the first incident case of an event as a relevant outcome, which has been shown to be a valid approach. ${ }^{10}$

Secondly, the occurrence of an event should not alter the probability of subsequent use. For example, if the event is a contraindication to prescribing the drug of interest, it would lead to a low rate of events in the period leading up to the first period of use and may artificially exaggerate the relative rate of events occurring in exposed versus unexposed periods. This potential bias can be overcome by removing a predefined period of time before exposure from all other unexposed (baseline) time. $^{12}$
Thirdly, the event of interest must not censor the observation period-for example, if the event increases the likelihood of death-although there is some evidence that the method is robust to this assumption. ${ }^{11}$ Whether this assumption is fulfilled can be readily checked by measuring short term mortality after the event of interest.

\section{Study participants, exposure, and outcome}

Patients of all ages were selected from the population registered with the Clinical Practice Research Datalink with an active registration status at any point during the study period, between 1 January 1999 and 30 March 2011. We chose 1999 as the earliest possible start date as orlistat was launched that year. Eligible participants were patients with both the exposure of interest (prescribed orlistat) and the outcome of interest (idiopathic acute liver injury) during this period. To ensure that we included only new users of orlistat, all participants had to have at least 12 months continuous registration in the Clinical Practice Research Datalink before the first recorded orlistat prescription; the first 12 months of continuous registration were excluded from all analyses. The end of observation for each participant was the earliest of death, transfer out of the practice, last data collection date for the practice, or the date a medical code was recorded that excluded the patient from further observation. Within this cohort of exposed patients, we identified cases by a search of data in both Clinical Practice Research Datalink and Hospital Episode Statistics, using Read codes, test results, and ICD-10 codes.

The specific nature of any hepatic effect of orlistat has not been established and for this reason we adopted a broad case definition. We defined patients as cases if they had a first recorded incident idiopathic liver event during continuous registration, again excluding the first 12 months of continuous registration to ensure events were incident. We defined cases as "definite" if they had a relevant clinical code (Read codes in the Clinical Practice Research Datalink and ICD-10 codes in Hospital Episode Statistics) indicating a possible idiopathic hepatic event plus evidence of abnormal liver function test results within 30 days plus a referral to hospital within 60 days related to a hepatic event. The incident date for the case was set as the earliest of these events. We defined "probable" cases as those with a relevant medical code but with only one of the further "definite" case criteria-for example, a record of a referral or a confirmatory liver function test result.

The definition of an abnormal liver function test result indicative of an adverse hepatic event was either an increase of more than twice the upper limit of normal for alanine aminotransferase level or a combined increase in aspartate aminotransferase, alkaline phosphatase, and total bilirubin levels, provided one of them was twice the upper limit of normal. Because the first adverse liver event could affect the likelihood of subsequent events, we only used the first incident occurrence of idiopathic acute liver injury in the analysis. ${ }^{10}$

The primary study population comprised both definite and probable cases. The distinction between definite and probable is to some extent driven by the severity of events, with less severe events possibly not resulting in a secondary referral. The analysis of the combined group therefore maximises statistical power. We also carried out a restricted analysis among patients who were classified as definite cases, as they had additional evidence of liver injury.

We used recorded event terms indicating other likely causes of the hepatic injury to exclude patients from the study population. These were applied in three categories. Firstly, we excluded all 
patients with a history of permanent liver damage (for example, cirrhosis) before the event date. Secondly, we excluded patients with a record of any cancer before the event or up to 12 months after the event. Finally, we excluded patients with a record of other non-drug related hepatic illness or major risk for hepatic illness (for example, viral hepatitis, non-alcoholic fatty liver, alcoholism) up to 12 months before or after the event. For any patients who developed one of these conditions more than 12 months after their incident case event, we censored follow-up at the time the condition was recorded.

Orlistat use was determined using prescribing records in the Clinical Practice Research Datalink. The expected duration of each prescription was calculated using dosage and pack size information where available. Where this information was missing, we imputed the median pack duration of 28 days. Where a gap of less than 60 days occurred between the end of one prescription and the start of another, we considered use to be continuous to allow for lack of adherence and drug stockpiling.

\section{Statistical analysis}

We used conditional poisson regression to calculate incidence rate ratios comparing the risk of liver injury during periods of orlistat use with periods of non-use, adjusting for age in five year bands. Figure $1 \Downarrow$ shows a typical timeline for a single patient in the study. We divided patient time into six discrete categories; absence of orlistat, 90 days before first ever orlistat prescription, first 30 days of orlistat use, 31-60 days of orlistat use, 61-90 days of orlistat use, and more than 90 days of orlistat use. We included the 90 day period before first orlistat use as a precaution to assess whether the likelihood of starting orlistat is temporarily affected by having a liver event. The removal of this period from baseline time avoids biased estimates when such a mechanism is present. ${ }^{10}$ We separated the first 90 days of use into three 30 day periods to allow the detection of any temporary change in the relative risk of liver events; if a causal association with treatment exists it is possible that the risk of hepatic events may vary with duration of use, and many adverse drug reactions occur during the early stages of a treatment.

\section{Additional analyses}

We carried out several sensitivity and secondary analyses. Firstly, we censored all follow-up at 1 January 2009 as orlistat was made available without prescription during 2009 and so ascertainment of use beyond this time may be incomplete. An additional restricted analysis was done excluding all Hospital Episode Statistics data, since data from this linkage were not available for all patients. After observing an unexpected finding in the 90 day period before first use, we did a further analysis comparing the risk of liver events in the first 90 or 30 days of treatment with that in the 90 or 30 days before first orlistat use. Finally, to check the assumption that events do not lead to censoring of the observation period, we also determined the number of deaths within one year of the recorded liver injury.

All data analysis was carried out using Stata 12.

\section{Results}

The Clinical Practice Research Datalink contained records for 94695 patients who had received at least one prescription for Orlistat. Of these, 2973 patients had an eligible clinical code indicating possible liver injury. Overall, 1985 were excluded; 998 had their index event outside the observation period and 447 had recorded events that met the exclusion criteria. A further
272 were excluded as they were found to have normal liver function test results and 268 had no liver function test results recorded and were not referred to secondary care. This left 988 eligible cases. Figure $2 \Downarrow$ shows the exclusion and case categorisation process.

Table $1 \Downarrow$ shows the personal characteristics of the definite and possible cases. Among the 988 definite and probable cases, the median age at first orlistat prescription was 48 years and the mean observation period was 9.2 years. The mean duration of orlistat treatment was 10.8 months, and this was slightly longer for women than for men. Ninety four patients $(9.5 \%)$ had a liver event during orlistat treatment, with the remaining 894 occurring during periods of non-use. Age and sex patterns were similar when restricted to the 335 definite cases, with $27(8 \%)$ experiencing a liver event during orlistat use.

Table $2 \Downarrow$ summarises the hepatic events that identified patients as cases. Most were coded as raised liver function test results $(85 \%)$, with a small proportion indentified through codes for jaundice $(14 \%)$, hepatitis $(2 \%)$, or liver failure $(<1 \%)$.

Table $3 \Downarrow$ shows age adjusted incidence rate ratios for liver injury comparing the predefined risk periods of orlistat use with periods of non-use. For the 988 definite and probable cases the risk of liver injury was more than doubled during the first 30 days of treatment (incidence rate ratio 2.21, 95\% confidence interval 1.43 to 3.42 ). This decreased to 1.06 (0.57 to 1.99 ) during treatment days 31-60. The risk was not increased during treatment days $61-90(1.32,0.75$ to 2.34$)$ and $>90(0.78,0.58$ to 1.05$)$. The incidence rate ratio was also increased during the 90 day period before the first orlistat prescription $(1.50,1.10$ to 2.06). This unexpected finding led us to undertake a retrospective secondary analysis comparing the incidence of liver injury in the 90 days before the first orlistat prescription with the 90 day period after the first prescription. In this analysis, we found no evidence that the risk of liver injury was higher in the period after treatment had started than in the period before treatment $(1.02,0.67$ to 1.56$)$. The incidence rate ratio with the shorter period of 30 days before and after a first prescription for orlistat was 1.11 (0.59 to 2.06).

In the analysis restricted to definite cases, an increased risk of liver injury was seen in the 90 days before orlistat prescription $(1.75,1.05$ to 2.91$)$. Evidence of an increased risk during any period of orlistat use was lacking, although the number of events in each exposure period was low. Comparing the 90 day period after the first orlistat prescription with the 90 day period before the prescription the incidence rate ratio was 0.63 (0.28 to 1.38 ), again suggesting no evidence of an increased risk during early use.

Sensitivity analyses censoring all follow-up at 1 January 2009 (orlistat became available without prescription in the United Kingdom in 2009) or excluding Hospital Episode Statistics data from the analysis made no material difference to the results. Of note, Hospital Episode Statistics data only informed the case status for 52 cases. Mortality within a year of the liver injury was low, with six patients recorded as dead within 12 months of the event. An analysis excluding these patients had no impact on the results.

\section{Discussion}

The incidence of acute liver injury from orlistat use is approximately doubled during a short period both before $(90$ days) and after the start of treatment (30 days) compared with background incidence, suggesting that the start of treatment tends to coincide with a period of increased risk of liver injury. Our results were not suggestive of a causal link between orlistat 
use and liver injury: the incidence just after starting treatment was comparable with that just before starting treatment. The incidence of liver injury after more than 30 days use was similar to that during the period of non-use. These results are likely to be generalisable to other populations receiving orlistat, as the Clinical Practice Research Datalink used in this study is representative of the UK population.

Several factors may explain why the start of orlistat treatment tends to coincide with times of increased risk of liver injury.

The initiation of any new drug often occurs at a time of specific concerns about a patient's health. For an obese patient, the decision to start orlistat may be a response to changes in obesity related health problems. This could include symptoms related to underlying liver disease, which is a common complication of obesity, ${ }^{13}$ and the investigation of such symptoms may result in a diagnosis that would qualify a patient as a case in our study. Even changes in health status not directly related to the liver could result in a range of tests being performed, including liver function tests; again the results of these could lead to the identification of asymptomatic increases in liver function markers sufficient to qualify the patient as a case. Consistent with this hypothesis, we noted that the rate of liver function testing (regardless of result) among all 94695 patients receiving orlistat was higher during the 90 days before and after the first prescription for orlistat (774 tests per 1000 patient years) compared with other periods (481 tests per 1000 patient years). We chose to use the self controlled case series design because participants act as their own controls, and therefore some of the problems of confounding inherent to other study designs are avoided. Treated and untreated patients generally differ in important ways that can influence the outcome being studied, and although methods to account for these differences are routinely used in observational research, residual confounding often remains a problem. The key assumptions underpinning the self controlled case series were met, making it a good choice of design for this study; the event of interest was non-recurrent and did not lead to any substantial increase in short term mortality. Although our results show that the event itself may lead to a short term change in the likelihood of receiving orlistat, this period of influence seems to be short lived and was accounted for in our analysis by separating out a period before orlistat use. The comparison of later usage with non-usage periods was consistent with no change in event rate, and thus a constant risk of liver injury during all other times. The separation of the period before orlistat use from all other periods of non-use also highlights a unique and important advantage of the self controlled case series over the cohort method as it allowed us to directly compare the risk of liver injury in the period before orlistat use with the first period of use. In a traditional cohort design, the pre-use period would not be included in the analysis, and could have led to the conclusion that starting orlistat treatment is associated with an increased risk of liver injury.

\section{Comparison with other studies}

These results need to be viewed in the context of other information already available on this topic. Pre-marketing randomised trials did not detect liver injury as a possible effect of orlistat, and a recent meta-analysis including around 10000 patients from randomised trials found no association between orlistat and increases in either alanine aminotransferase or bilirubin levels. ${ }^{5}{ }^{14}$ However, the selective nature and limited numbers included in clinical trials can mean important safety findings are not identified. Formal studies of this issue based on real world usage of orlistat have not been published to date and the signal regarding possible liver injury is based on the analysis of individual case reports submitted to regulatory authorities. Although such analyses can sometimes provide useful insight into possible causality, such conclusions are often not possible. Indeed our study suggests one of the reasons individual doctors may have made a link between orlistat and adverse liver events results from a non-causal and temporary increase in the likelihood of such events around this time.

\section{Strengths and limitations of this study}

For this study we defined the outcome by searching electronic general practice records for evidence of liver injury. Where available we supplemented this information with records of hospital admissions, although most cases $(95 \%)$ were identified from general practice records only. This could be because the severity of liver injury in these patients was generally insufficient to warrant admission to hospital.

We graded cases as either definite or probable depending on the evidence available, although the less severe nature of many events is likely to mean some definite cases were incorrectly classed as probable. The impact of such misclassification is likely to be minimal as the results of the analysis of only definite cases also failed to find evidence of an increased risk associated with orlistat. The accuracy of event timing is important to ensure events are counted during the correct usage time. While we have taken care to assign incidence at the earliest date with evidence of liver injury, it is possible that some events could have started earlier. However, this is unlikely to have led to large discrepancies in event timing, and if an event had been caused by orlistat it would generally have been assigned to a later period of treatment, meaning an association would still be detected.

Notably, in this study most events identified were of raised liver function test results, with few cases of serious liver injury such as hepatic failure or necrosis. Of the events occurring during orlistat treatment $99 \%$ were of raised liver function test results or jaundice, with a single case of hepatitis and no cases of liver failure or necrosis. Serious liver events were therefore rare during treatment with orlistat in this group of almost 100000 patients. Although it is possible that low level asymptomatic changes in liver function may have gone undetected, more severe events would almost certainly require medical attention and would be recorded by the general practitioner.

We defined the patients' usage status according to date of prescription issue, rather than precise records of drug consumption, which are not available. It is likely that in some instances use will have been overestimated, which could lead to a result bias towards no effect. However, the median period of usage was almost a year, and it is unlikely that patients continued to obtain repeat prescriptions for a drug they were not using.

Other possible biases related to orlistat being obtained without prescription after 2009 or case status being ascertained differently in patients from practices linked to Hospital Episode Statistics were dealt with in sensitivity analyses. The results were robust to these possible problems.

\section{Conclusions}

Obesity has become a major health problem worldwide and orlistat is currently the only available drug intervention of proved efficacy. Possible safety concerns about orlistat are therefore of great importance. By using the self controlled case series design we were able to establish that the risk of liver injury was increased to a similar degree both just before and just after starting treatment, strongly suggesting that the association is non-causal. Our results imply that studies based 
only on traditional epidemiological designs or spontaneous adverse events are likely to detect associations between starting orlistat treatment and liver injury, but such associations should not be interpreted as evidence for an adverse causal effect of the drug unless an increased risk in the period immediately before the start of treatment can be excluded.

Contributors: IJD conceived and designed the study, analysed the data, interpreted the results, and cowrote the paper. JL and RB analysed the data, interpreted the results, and cowrote the paper. KB and LS designed the study, interpreted the results, and cowrote the paper. IJD acts as guarantor.

Competing interests: All authors have completed the ICMJE uniform disclosure form at www.icmje.org/coi_disclosure.pdf (available from the corresponding author) and declare: IJD is funded by a Medical Research Council methodology fellowship, KB is funded by a National Institute for Health Research postdoctoral fellowship, and LS is funded by a Wellcome Trust fellowship; IJD holds stock in GlaxoSmithKline and consults for GlaxoSmithKline, Takeda, and Gilead on topics not related to orlistat; LS consults for GlaxoSmithKline on topics not related to orlistat; and no other relationships or activities that could appear to have influenced the submitted work. The funders played no role in the design and conduct of the study; collection, management, analysis, and interpretation of the data; and preparation, review, or approval of the manuscript.

Ethical approval: This study was approved by the independent scientific advisory committee for Medicines and Healthcare products Regulatory Agency database research and the London School of Hygiene and Tropical Medicine ethics committee.

Data sharing: No additional data available.
1 Guerciolini R. Mode of action of orlistat. Int J Obes Relat Metab Disord 1997;21(Suppl 3):S12-23.

2 Food and Drug Administration. Orlistat (marketed as Alli and Xenical): early communication about an ongoing safety review. FDA, 2009. 2012. www.fda.gov/Safety/MedWatch/ SafetyInformation/SafetyAlertsforHumanMedicalProducts/ucm180025.htm.

3 Food and Drug Administration. Drug safety communication: completed safety review of Xenical/Alli (orlistat) and severe liver injury. FDA, 2010. 2012. www.fda.gov/Drugs/ DrugSafety/PostmarketDrugSafetylnformationforPatientsandProviders/ucm213038.htm.

4 European Medicines Agency confirms positive benefit-risk balance of orlistat-containing medicines. 2012. www.ema.europa.eu/docs/en_GB/document_library/Press_release/ 2012/02/WC500122878.pdf.

5 Morris M, Lane P, Lee K, Parks D. An integrated analysis of liver safety data from orlistat clinical trials. Obes Facts 2012;5:485-94. doi:10.1159/000341589. Published online 23 Jul 2012.

6 Lawson DH, Sherman V, Hollowell J. The General Practice Research Database. Q J Med 1998;91:445-52

7 García Rodríguez LA, Pérez Gutthann S. Use of the UK General Practice Research Database for pharmacoepidemiology. Br J Clin Pharmacol 1998;45:419-25.

8 Office for National Statistics. Key health statistics from general practice 1998. Office for National Statistics, 2000:149. Series MB6 No.2.

9 Hospital Episode Statistics. Hospital Episode Statistics Online. 2012. www.hesonline.nhs. $\mathrm{uk} /$.

10 Whitaker HJ, Farrington CP, Spiessens B, Musonda P. Tutorial in biostatistics: the self-controlled case series method. Stat Med 2006;25:1768-97.

11 Whitaker HJ, Hocine M, Farrington CP. The methodology of self-controlled case series studies. Stat Methods Med Res 2009;18:7-26.

12 Pratt NL, Roughead EE, Ramsay E, Salter A, Ryan P. Risk of hospitalization for stroke associated with antipsychotic use in the elderly. Drugs Aging 2010;27:885-93

13 Diehl AM. Hepatic complications of obesity. Gastroenterol Clin North Am 2010;39:57-68.

14 Food and Drug Administration. Questions and answers: Orlistat and severe liver injury. FDA, 2010. 2012. www.fda.gov/Drugs/DrugSafety/

PostmarketDrugSafetyInformationforPatientsandProviders/ucm213040.htm

\section{Accepted: 8 March 2013}

\section{Cite this as: BMJ 2013;346:f1936}

This is an Open Access article distributed in accordance with the Creative Commons Attribution Non Commercial (CC BY-NC 3.0) license, which permits others to distribute, remix, adapt, build upon this work non-commercially, and license their derivative works on different terms, provided the original work is properly cited and the use is non-commercial. See: http://creativecommons.org/licenses/by-nc/3.0/. 


\section{What is already known on this topic}

Since early 2000 reports of liver injury associated with orlistat have accumulated, raising concerns about its safety Although randomised trials found no such association, observational studies based on real world orlistat use are lacking

\section{What this study adds}

In a large population based cohort, the rate of adverse liver events seems to be temporarily increased both immediately before and immediately after starting treatment with orlistat

This suggests that the increased risk of liver injury linked to orlistat may reflect changes in underlying health status associated with the decision to start treatment, rather than a causal effect of the drug

\section{Tables}

\section{Table 1 | Characteristics of study population}

\begin{tabular}{|c|c|c|c|c|c|c|}
\hline \multirow[b]{2}{*}{ Characteristics } & \multirow[b]{2}{*}{$\begin{array}{l}\text { No of } \\
\text { patients }\end{array}$} & \multirow[b]{2}{*}{$\begin{array}{l}\text { Mean (SD) age at start } \\
\text { of orlistat (years) }\end{array}$} & \multicolumn{2}{|c|}{ Unexposed period* } & \multicolumn{2}{|r|}{ Exposed period } \\
\hline & & & No of events & $\begin{array}{l}\text { Mean (SD) duration of } \\
\text { follow-up (years) }\end{array}$ & No of events & $\begin{array}{l}\text { Mean (SD) duration of use } \\
\text { (years) }\end{array}$ \\
\hline
\end{tabular}

Definite and probable

cases:

\begin{tabular}{lcccccc}
\hline All & 988 & $48.4(12.8)$ & 894 & $9.2(2.7)$ & 94 & $0.9(0.9)$ \\
\hline Men & 309 & $48.9(11.8)$ & 284 & $9.1(2.8)$ & 25 & $0.8(0.8)$ \\
\hline Women & 679 & $48.3(13.3)$ & 610 & $9.3(2.7)$ & 69 & $0.9(1.0)$ \\
\hline Definite cases: & & & & & \\
\hline All & 335 & $47.9(13.1)$ & 308 & $9.3(2.5)$ & 27 & $0.9(0.9)$ \\
\hline Men & 98 & $48.1(11.1)$ & 89 & $9.0(2.4)$ & 9 & $0.8(0.7)$ \\
\hline Women & 237 & $47.8(13.9)$ & 219 & $9.4(2.6)$ & 18 & $0.9(1.0)$ \\
\hline
\end{tabular}

*Before or after orlistat use. 
Table 2| Distribution of adverse liver events. Values are numbers (percentages)

\begin{tabular}{|c|c|c|c|}
\hline Recorded hepatic event & Definite cases & Probable cases & Total \\
\hline Raised liver function test results & $279(83)$ & $557(85)$ & $836(85)$ \\
\hline Jaundice & $53(16)$ & $83(13)$ & $136(14)$ \\
\hline Hepatitis & $7(2)$ & $12(2)$ & $19(2)$ \\
\hline Liver failure & 0 & $1(<1)$ & $1(<1)$ \\
\hline Liver necrosis & 0 & 0 & 0 \\
\hline Other* & $5(1)$ & $8(1)$ & $13(1)$ \\
\hline
\end{tabular}

Patients with more than one recorded hepatic event during same episode are included in all relevant categories, therefore counts cannot be summed to equal total patients.

*Mostly procedural—for example, liver biopsy. 
Table 3/ Self controlled case series analysis for orlistat use and risk of liver injury

Variables

Patient years No of events Age adjusted rate ratio $(95 \% \mathrm{Cl})$

Definite and probable cases $(n=988)$

Primary analyses:

\begin{tabular}{lccc}
\hline Absence of orlistat & 8872 & 852 & - \\
\hline 90 days before prescription & 241 & 42 & $1.50(1.10$ to 2.06$)$ \\
\hline 1-30 days orlistat & 81 & 21 & $2.21(1.43$ to 3.42$)$ \\
\hline 31-60 days orlistat & 80 & 10 & $1.06(0.57$ to 1.99$)$ \\
\hline $61-90$ days orlistat & 78 & 12 & $1.32(0.75$ to 2.34$)$ \\
\hline$>90$ days orlistat & 986 & 51 & $0.78(0.58$ to 1.05$)$
\end{tabular}

Secondary analyses:

\begin{tabular}{lccc}
\hline 90 days before prescription & 241 & 42 & - \\
\hline $1-90$ days orlistat & 240 & 43 & $1.02(0.67$ to 1.56$)$ \\
\hline 30 days before prescription & 81 & 19 & - \\
\hline $1-30$ days orlistat & 81 & 21 & $1.11(0.59$ to 2.06$)$ \\
\hline
\end{tabular}

\section{Definite cases $(n=335)$}

Primary analyses:

\begin{tabular}{lccc}
\hline Absence of orlistat & 3042 & 292 & - \\
\hline 90 days before prescription & 82 & 16 & $1.75(1.05$ to 2.91$)$ \\
\hline 1-30 days orlistat & 27 & 1 & $0.32(0.05$ to 2.30$)$ \\
\hline 31-60 days orlistat & 27 & 3 & $0.97(0.31$ to 3.04$)$ \\
\hline $61-90$ days orlistat & 26 & 6 & $2.02(0.90$ to 4.54$)$ \\
\hline$>90$ days orlistat & 211 & 17 & $0.72(0.43$ to 1.22$)$ \\
\hline Secondary analyses: & & & \\
\hline 90 days before prescription & 82 & 16 & $0.63(0.28$ to 1.38$)$ \\
\hline 1-90 days orlistat & 81 & 10 & - \\
\hline 30 days before prescription & 27 & 5 & $0.20(0.02$ to 1.71$)$ \\
\hline 1-30 days orlistat & 27 & 1 &
\end{tabular}




\section{Figures}

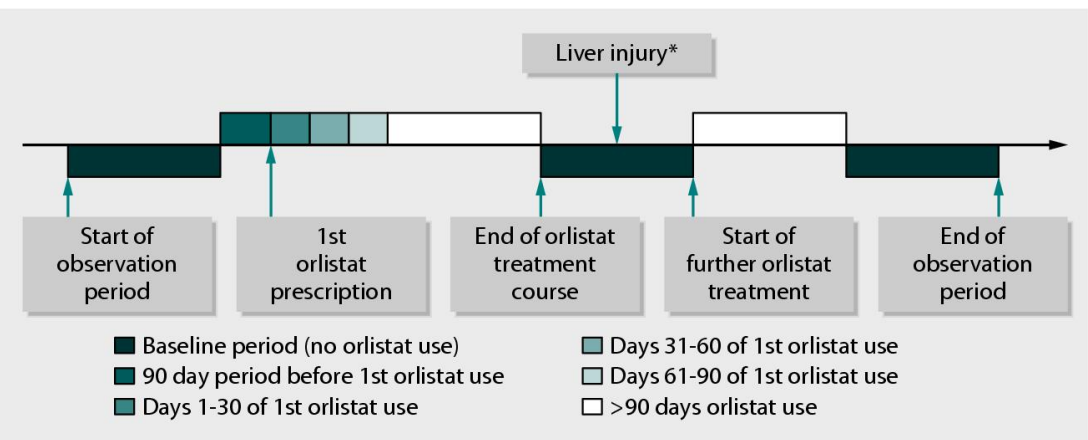

Fig 1 Typical timeline for patient in study. *Liver injury could occur at any point during observation period

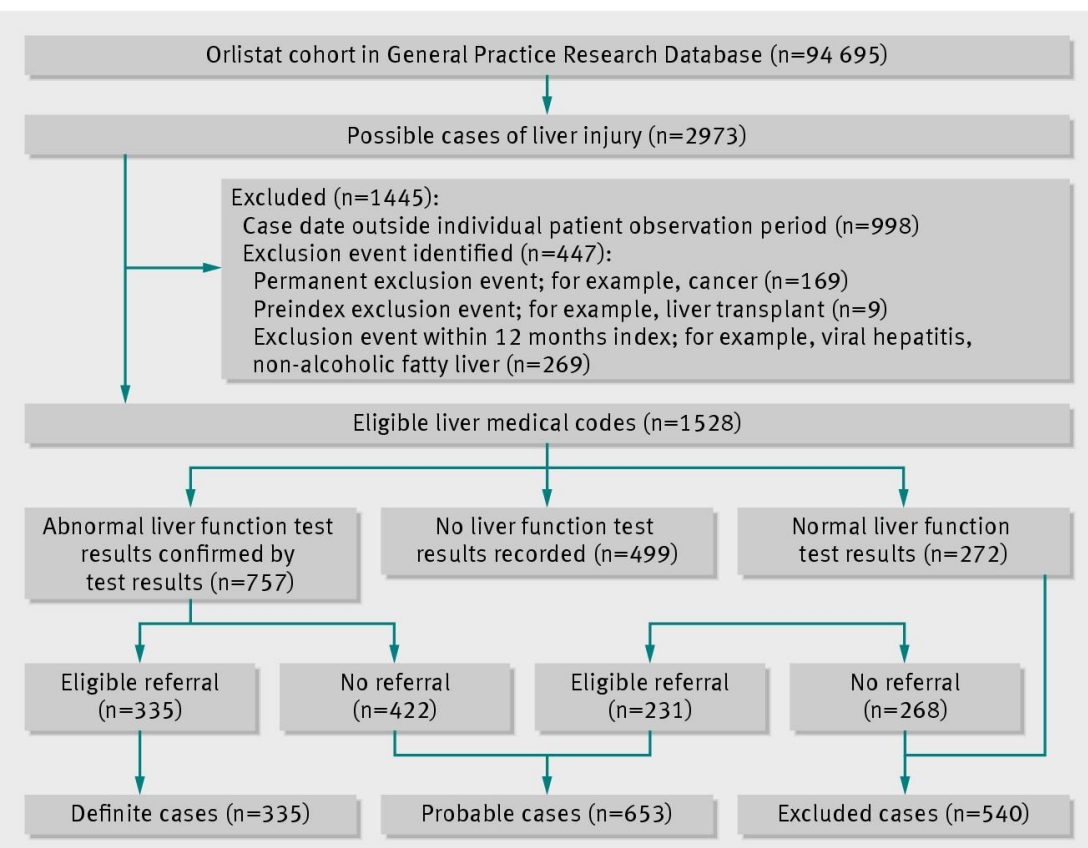

Fig 2 Flow of participants through study 